\title{
Periodicity of Generalized Fibonacci-like Sequences
}

\author{
Alexander V. Evako* \\ "Dianet”, Laboratory of Digital Technologies, Moscow, Russia \\ *Corresponding author: evakoa@mail.ru
}

\begin{abstract}
Generalized Fibonacci-like sequences appear in finite difference approximations of the Partial Differential Equations based upon replacing partial differential equations by finite difference equations. This paper studies properties of the generalized Fibonacci-like sequence $F_{n+2}=A+B F_{n+1}+C F_{n}$. It is shown that this sequence is periodic with period $\mathrm{T}>2$, if $\mathrm{C}=-1,|\mathrm{~B}|<2$.
\end{abstract}

Keywords: generalized fibonacci-like sequence, periodicity, discrete dynamical system, PDE, graph, network, digital space

Cite This Article: Alexander V. Evako, "Periodicity of Generalized Fibonacci-like Sequences." Applied Mathematics and Physics, vol. 5, no. 1 (2017): 11-18. doi: 10.12691/amp-5-1-2.

\section{Introduction}

Fibonacci sequences have attracted attention of scientists from different fields. Fibonacci numbers are widely used in many areas from applied mathematics to physics. The Fibonacci sequence, Lucas sequence, Pell sequence and periodic generalized Fibonacci sequences modulo $m$ have been intensively studied for many years. Over the last decades, numerous useful theoretical results have been obtained in this field (see, e.g., $[1,10,15,17,21,22]$ ).

In this paper, we introduce and study a new generalization of Fibonacci-like sequences; we are interested in the periodicity of the Fibonacci-like sequence.

The essential fact is that finite difference PDE equations on discrete domains contain generalized Fibonacci-like sequences as basic elements of these equations, and knowledge of features of these sequences plays an important role in analyzing properties computational solutions of PDE such as the period and the frequency of oscillations.

Finite difference approximations of the PDE are based upon replacing partial differential equations by finite difference equations $[13,18]$. In the past decades, computational solutions of PDE on various domains including non-orientable surfaces such as a Moebius strip and a Klein bottle have been studied by scientists from different fields. The study was derived from obvious practical and science background. In physics, a considerable interest has emerged in studying lattice models on non-orientable surfaces as new challenging unsolved lattice-statistical problems and as a realization and testing of predictions of the conformal field theory (see, e.g., [12]). Many important technical and physical properties of non-orientable structural elements can be described by solutions of partial differential equations (PDE), where a non-orientable space serves as a domain. A problem exists in description of electronic and nuclear motions in nano-technology structures and biological networks.
Modeling blood flow through a capillary network or road traffic requires a system of differential equations on a graph or network. Since analytic solutions of PDE can be obtained only in simple geometric regions, for practical problems, it is more reasonable to use computational or numerical solutions. It can be done by implementing as domains graphs, networks and digital spaces, which are discreet counterparts of continuous spaces, and by transferring PDE from a continuous area into discrete one. A review of works devoted to partial differential equations on networks and graphs can be found in $[4,14,16]$.

The material to be presented in Section 2 begins with the investigation of properties of a periodic continuous function $f(x)$, where $f(x+1)=A+B f(x)+C f(x-1)$. It is shown that if $f(x)$ is periodic with period $T>2$ then $|B|<2, C=-1$.

In section 3 , we study properties of the sequence, which is defined by recurrence relation $F_{n+2}=A+B F_{n+1}+$ $C F_{n}$ with $F_{0}=a, F_{1}=b, n \geq 0$ where A, B C, $a$ and $b$ are real numbers. We show that if $|B|<2, C=-1$ then $\left\{F_{n}\right\}$ is periodic with the period $T>2$.

Section 4 presents numerical examples of periodic Fibonacci-like sequences and gives examples of application of Fibonacci-like sequences to the numerical solutions of the wave equation on the graph containing two vertices and on the graph containing sixteen vertices and presenting a non-orientable Klein bottle, which is an important object of study in physics. Using properties of Fibonacci-like sequences, we show that the period of oscillations on the two-point graph is $\mathrm{T}=13.9$. Oscillations at different points of the Klein bottle are also periodic.

\section{Periodicity of Fibonacci-like Continuous Functions}

In a numerical solution of the one-dimensional wave equation by the finite difference method, the spatial domain and the temporal domain are replaced by a set of mesh points, the second-order derivatives can be replaced 
by central differences $[4,13,14,16]$. The solution of the wave equation is replaced by the mesh function, which approximates the exact solution at the mesh points. The obtained discretization scheme has the form $u_{i}^{n+1}=2 u_{i}^{n}$ $+c^{2}\left(u_{i-1}^{n}-2 u_{i}^{n}+u_{i}^{n}\right)-u_{i}^{n-1}$, where $c=k(\Delta t / \Delta x)$.

Note that the solution is periodic in time. Using this formula, consider properties of periodic continuous function $\mathrm{f}(\mathrm{x})$ on domain $\mathrm{R}$ provided that $f(x+1)=A+$ $B f(x)+C f(x-1)$.

\section{Theorem 1}

Let $\mathrm{f}(\mathrm{x})$ be a continuous function on domain $\mathrm{R}$, and let

$$
f(x+1)=A+B f(x)+C f(x-1)
$$

for any $x \in R$. If $\mathrm{f}(\mathrm{x})$ is a periodic function with period $T>2$ then $|B|<2, C=-1, T=\frac{2 \pi}{\omega}, \omega=\arccos \frac{B}{2}$.

Proof.

Let $\mathrm{f}(\mathrm{x})$ is periodic with period $T=2 \pi / \omega$. Consider the Fourier series representing $\mathrm{f}(\mathrm{x})$.

$$
f(x)=a_{0}+\sum_{n=1}^{\infty} a_{n} \cos \omega n x+b_{n} \sin \omega n x .
$$

Using (2), it is easy to see that

$$
\begin{aligned}
& f(x+1)-A-B f(x)-C f(x-1) \\
& =c_{0}+\sum_{n=1}^{\infty} c_{n} \cos \omega n x+d_{n} \sin \omega n x,
\end{aligned}
$$

where

$$
\begin{gathered}
c_{0}=a_{0}-C a_{0}-B a_{0}-A \\
c_{n}=a_{n}(\cos \omega n-C \cos \omega n-B)+b_{n}(1+C) \sin \omega n \\
d_{n}=-a_{n}(1+C) \sin \omega n+b_{n}(\cos \omega n-C \cos \omega n-B) .
\end{gathered}
$$

Since

$$
f(x+1)-A-B f(x)-C f(x-1)=0
$$

for $\forall x$ then for any $\mathrm{n}$, coefficients $\mathrm{c}_{0}, \mathrm{c}_{\mathrm{n}}$ and $\mathrm{d}_{\mathrm{n}}$ are equal to zero.

$$
c_{0}=c_{n}=d_{n} \equiv 0 .
$$

Let $\mathrm{n}=1$.

$$
\begin{aligned}
c_{1}= & a_{1}(\cos \omega-C \cos \omega-B) \\
& +b_{1}(1+C) \sin \omega=0 \\
d_{1}= & -a_{1}(1+C) \sin \omega \\
& +b_{1}(\cos \omega-C \cos \omega-B)=0 .
\end{aligned}
$$

As $\mathrm{f}(\mathrm{x})$ is periodic with basic period $T=\frac{2 \pi}{\omega}$ then $a_{1} \neq 0$ and /or $b_{1} \neq 0$, which means that

$$
\left\{\begin{array}{c}
\cos \omega-C \cos \omega-B=0 \\
(1+C) \sin \omega=0
\end{array}\right.
$$

Suppose that $\sin \omega \neq 0$, i.e., $\omega \neq k \pi, T \neq \frac{2}{k}$. It follows from (7) that $1+C=0, \cos \omega=\frac{B}{1-C},|\cos \omega|<1$. Therefore, $C=-1, B=2 \cos \omega,|B|<2$. Hence,

$$
\omega=\arccos \frac{B}{2} \text { and } T=\frac{2 \pi}{\omega} .
$$

Let $\mathrm{n}>1$.

$\left.c_{n}=a_{n}(\cos \omega n-C \cos \omega n-B)+b_{n}(1+C) \sin \omega n=0\right)$

$d_{n}=-a_{n}(1+C) \sin \omega n+b_{n}(\cos \omega n-C \cos \omega n-B)=0$.

For the same reason as above, if $\cos \omega n-C \cos \omega n-$ $B \neq 0$ then $a_{n}=b_{n} \equiv 0$, if $\cos \omega n-C \cos \omega n-B=0$ then $a_{1} \neq 0$ and/or $b_{1} \neq 0$. Note that $\cos \omega n-$ $C \cos \omega n-B=0$ if $(n \pm 1) \omega=2 \pi k, n, k \in N$.

Finally, find $a_{0}$ from equation (3). Notice that $C=-1$, $|B|<2$.

$$
a_{0}=\frac{A}{1-C-B}=\frac{A}{2-B} .
$$

Thus, if $|B|<2$ and $C=-1$ then $\mathrm{f}(\mathrm{x})$ is periodic with basic period $T=\frac{2 \pi}{\omega}$ and $\omega=\arccos \frac{B}{2}$.

$$
\begin{aligned}
f(x)= & a_{0}+a_{1} \cos \omega x+b_{1} \sin \omega x \\
& +\sum_{n \in G} a_{n} \cos \omega n x+b_{n} \sin \omega n x,
\end{aligned}
$$

where $n \in G$ if $(n \pm 1) \omega=2 \pi k, \quad n, k \in N$. This completes the proof.

\section{Theorem 2}

Let $\mathrm{f}(\mathrm{x})$ be a continuous periodic function on domain $\mathrm{R}$ with period, $T=\frac{2}{k}, k \in N, k \neq 0$ and for any $x \in R$,

$$
f(x+1)=A+B f(x)+C f(x-1) .
$$

Then $f(x+1)=f(x)$ if $k=2 n$.

$f(x+1)=D-f(x)$ if $k=2 n+1$.

\section{Proof}

Let $\mathrm{k}$ is even, i.e., $\mathrm{k}=2 \mathrm{n}$ and $T=\frac{1}{n}$. Then

$$
f(x+1)=f(x+n T)=f(x) .
$$

This means that in (9), $A=0, B=1$,

$$
C=0, f(x+1)=f(x) \text {. }
$$

Let $\mathrm{k}$ is odd, i.e., $\mathrm{k}=2 \mathrm{n}+1$ and $T=\frac{2}{2 n+1}, \omega=2 \pi n+$ $\pi$. Then $f(x+1)=f\left(x+\frac{T}{2}\right)$ and $f(x+1)=$ $f(x-1+(2 n+1) T)=f(x-1)$. Since $\quad \sin \omega=$ 0 , $\cos \omega=-1$, then by (7), $B=C-1$. Therefore,

$$
\left\{\begin{array}{c}
f(x+1)=A+B f(x)+C f(x+1) \\
B=C-1
\end{array} .\right.
$$

It follows from (10) that $f(x+1)=\frac{A}{1-C}-f(x)$ $=D-f(x)$. This completes the proof.

We now outline a correspondence between the number $\mathrm{B}$ and the period $\mathrm{T}$ of function $f(x)=A+B f(x-1)-$ $f(x-2)$, where $|B|<2$. Let's investigate this question graphically. The graph $T=T(B)=2 \pi\left(\arccos \frac{B}{2}\right)^{-1}$ is 
shown in Figure 1. Obviously, $\lim _{B \rightarrow 2} T=\infty$, $\lim _{B \rightarrow-2} T=2$.

\section{Periodic generalized Fibonacci-like Sequences}

Periodic generalized Fibonacci sequences modulo m have been studied in a number of papers (see e.g. $[9,11,19,21])$. In this section, we consider the periodic generalized Fibonacci sequence

$$
F_{n+2}=A+B F_{n+1}+C F_{n}, n \geq 0, F_{0}=a, F_{1}=b
$$

In sequence (11), $A, B, C, a$ and $b$ are real numbers. In particular, $A, B, C, a$ and $b$ can be integer. The results of this section are directly deduced from the previous section. The following theorems are a direct consequence of theorems 1 and 2.

\section{Theorem 3}

Let $F_{n+2}=A+B F_{n+1}+C F_{n}, n \geq 0, \quad F_{0}=a, F_{1}=b$, be a sequence of real numbers, $(\mathrm{A}, \mathrm{B}, \mathrm{C}, \mathrm{a}, \mathrm{b}) \in R$. If $|B|<2, C=-1$ then $\left\{F_{n}\right\}$ is a periodic sequence with period,

$$
T=\frac{2 \pi}{\omega}, \omega=\arccos \frac{B}{2} \cdot T>2
$$

Proof.

Consider the function $f(x+1)=A+B f(x)+$ $C f(x-1)$ where $f(0)=a, f(1)=b$. Then $f(n+2)=$ $F_{n+2}, f(n+1)=F_{n+1}, f(n)=F_{n}$ for $n \geq 0$.

According to theorem 1 , if $|B|<2, C=-1$, then $\mathrm{f}(\mathrm{x})$ is periodic with period $T=\frac{2 \pi}{\omega}, \omega=\arccos \frac{B}{2} . T>1$, $T \neq 2$. Therefore, sequence $\left\{F_{n}\right\}$ is also periodic with period $T=\frac{2 \pi}{\omega}$. This completes the proof.

Consider a special case when the period $\mathrm{T}=1,2$.

\section{Theorem 4}

Let $F_{n+2}=A+B F_{n+1}+C F_{n}, n \geq 0, F_{0}=a, F_{1}=b$, be a sequence of real numbers, $(\mathrm{A}, \mathrm{B}, \mathrm{C}, \mathrm{a}, \mathrm{b}) \in R$.

If $A=0, B=1, C=0$ then $\left\{F_{n}\right\}$ is a periodic sequence with period $T=1, F_{n+1}=F_{n}, \quad n \geq 0, F_{0}=F_{1}=a$.

If $C \neq 1, B=C-1$ then $\left\{F_{n}\right\}$ is a periodic sequence with period $T=2$,

$$
\begin{aligned}
& F_{n+1}=\frac{A}{1-C}-F_{n}=D-F_{n}, n \geq 0, \\
& F_{0}=a, F_{1}=\frac{A}{1-C}-a=D-a .
\end{aligned}
$$

Proof

Let $\mathrm{f}(\mathrm{x})$ be a continuous periodic function on domain $\mathrm{R}$, and $f(x+1)=A+B f(x)+C f(x-1)$.

If the period of $\mathrm{f}(\mathrm{x}) T=\frac{1}{k}, k \in Z$ then $f(x+1)=$ $f(x)$ by theorem 2 . Therefore, $F_{n+1}=F_{n}, n \geq 0, F_{0}=$ $F_{1}=a$.

If the period of $\mathrm{f}(\mathrm{x}) T=\frac{2}{2 k+1}, k \in Z$ then $f(x+1)=$ $f(x-1), \quad f(x+1)=\frac{A}{1-C}-f(x)=D-f(x), \quad f(1)=$ $\frac{A}{1-C}-f(0)=D-f(0)$ by theorem 2. Therefore,
$F_{n+1}=D-F_{n}, n \geq 0, F_{0}=a, F_{1}=D-a$

This

\section{Numerical Examples}

In this Section, we consider examples of the periodic generalized Fibonacci sequence and its applications to numerical solution of the wave equation on graphs.

\subsection{Numerical Examples of Periodic Fibonacci-like Sequences}

$F_{n+2}=A+B F_{n+1}+C F_{n}, n \geq 0, \quad F_{0}=a, F_{1}=b$ for $(\mathrm{A}, \mathrm{B}, \mathrm{C}, \mathrm{a}, \mathrm{b}) \in R$.

\section{Example 1}

First, consider the case, when coefficient $\mathrm{B}$ is close to 2 . Let $A=3, B=1.8, C=-1, F_{0}=1, F_{1}=5$. Then $F_{n+2}=3+1.8 F_{n+1}-F_{n}, n \geq 0$. In Figure 2, the sequence profile $\mathrm{F}_{\mathrm{n}}, F_{n+2}=3+1.8 F_{n+1}-F_{n}, n \geq 0$, is plotted for $n=1, \ldots 50$. The plot illustrates the periodicity of $\mathrm{F}_{\mathrm{n}}$ with period $T=13.93$, as it follows from formulas (12) $T=\frac{2 \pi}{\omega}, \omega=\operatorname{acos} \frac{B}{2}=\operatorname{acos} \frac{1.8}{2}$. For the first $n=0,1, \ldots 13$, the terms of $F_{n}$ are given in Table 1.

Table 1.

\begin{tabular}{|c|c|c|c|c|c|c|c|}
\hline $\mathrm{n}=$ & 0 & 1 & 2 & 3 & 4 & 5 & 6 \\
\hline $\mathrm{F}_{\mathrm{n}}=$ & 1.0 & 5.0 & 11.0 & 17.8 & 24.0 & 28.5 & 30.2 \\
\hline $\mathrm{n}=$ & 7 & 8 & 9 & 10 & 11 & 12 & 13 \\
\hline $\mathrm{F}_{\mathrm{n}}=$ & 28.9 & 24.8 & 18.8 & 12.0 & 5.8 & 1.4 & -0.2 \\
\hline
\end{tabular}

\section{Example 2}

In this case, take the following constants: $A=3, B=1$, $C=-1, F_{0}=1, F_{1}=5$. Then $F_{n+2}=3+F_{n+1}-$ $F_{n}, n \geq 0$. In Figure 3 , we have plotted the sequence profile $\mathrm{F}_{\mathrm{n}}, F_{n+2}=3+F_{n+1}-F_{n}, n \geq 0$, at $\mathrm{n}=1, \ldots 20$. The plot shows that $F_{n}$ is periodic with period $T=6$. According to (12), $\omega=\operatorname{acos} \frac{B}{2}=\operatorname{acos} \frac{1}{2}, T=\frac{2 \pi}{\omega}=6$. For the first $n=0,1, \ldots 13$, the terms of $F_{n}$ are shown in Table 2 .

Table 2

\begin{tabular}{|c|c|c|c|c|c|c|c|}
\hline $\mathrm{n}=$ & 0 & 1 & 2 & 3 & 4 & 5 & 6 \\
\hline $\mathrm{F}_{\mathrm{n}}=$ & 1.0 & 5.0 & 7.0 & 5.0 & 1.0 & -1.0 & 1.0 \\
\hline $\mathrm{n}=$ & 7 & 8 & 9 & 10 & 11 & 12 & 13 \\
\hline $\mathrm{F}_{\mathrm{n}}=$ & 5.0 & 7.0 & 5.0 & 1.0 & -1.0 & 1.0 & 5.0 \\
\hline
\end{tabular}

\section{Example 3}

Consider the case, when $A=3, B=0, C=-1, F_{0}=$ $1, F_{1}=5$. Then $F_{n+2}=3-F_{n}, n \geq 0$. In Figure 4 , the profile $\mathrm{F}_{\mathrm{n}}, F_{n+2}=3-F_{n}, n \geq 0$, is plotted for $\mathrm{n}=1, \ldots 20$. $F_{n}$ is periodic with period $T=4$, as it follows from (12). For the first $n=0,1, \ldots 13$, the terms of $F_{n}$ are given Table 3 .

Table 3

\begin{tabular}{|c|c|c|c|c|c|c|c|}
\hline $\mathrm{n}=$ & 0 & 1 & 2 & 3 & 4 & 5 & 6 \\
\hline $\mathrm{F}_{\mathrm{n}}=$ & 1.0 & 5.0 & 2.0 & -2.0 & 1.0 & 5.0 & 2.0 \\
\hline $\mathrm{n}=$ & 7 & 8 & 9 & 10 & 11 & 12 & 13 \\
\hline $\mathrm{F}_{\mathrm{n}}=$ & -2.0 & 1.0 & 5.0 & 2.0 & -2.0 & 1.0 & 5.0 \\
\hline
\end{tabular}




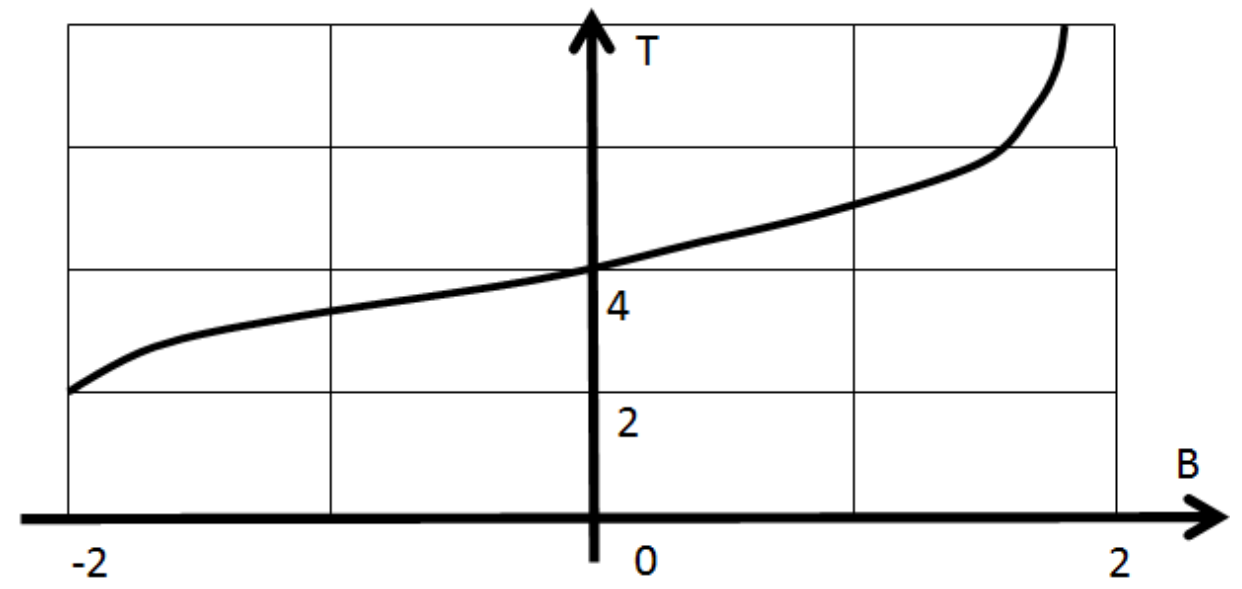

Figure 1. The period profile $\mathrm{T}$ of function $\mathrm{f}(\mathrm{x})$ and sequence $\mathrm{F}_{\mathrm{n}},|\mathrm{B}|<2$

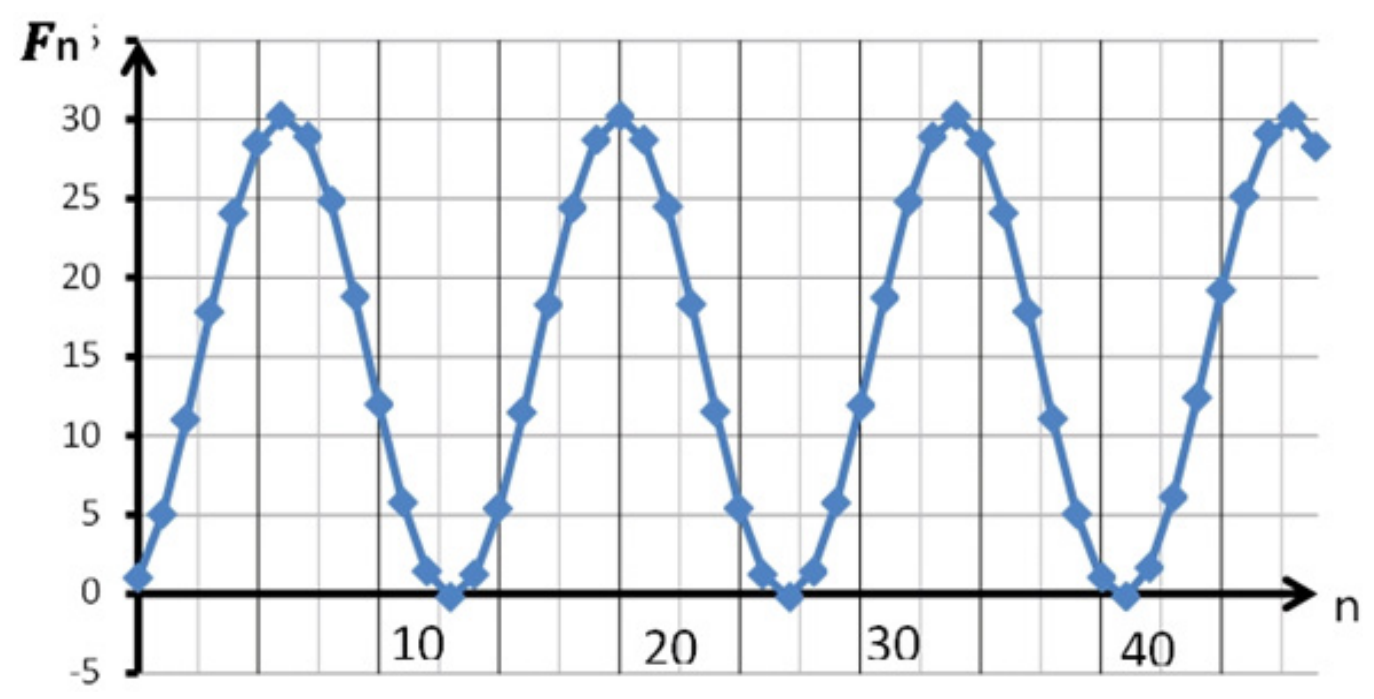

Figure 2. $\mathrm{F}_{\mathrm{n}}$ profile at $\mathrm{n}=0,1, \ldots 50 . F_{n+2}=3+1.8 F_{n+1}-F_{n}, n \geq 0, F_{0}=1, F_{1}=5 . T=13.93$

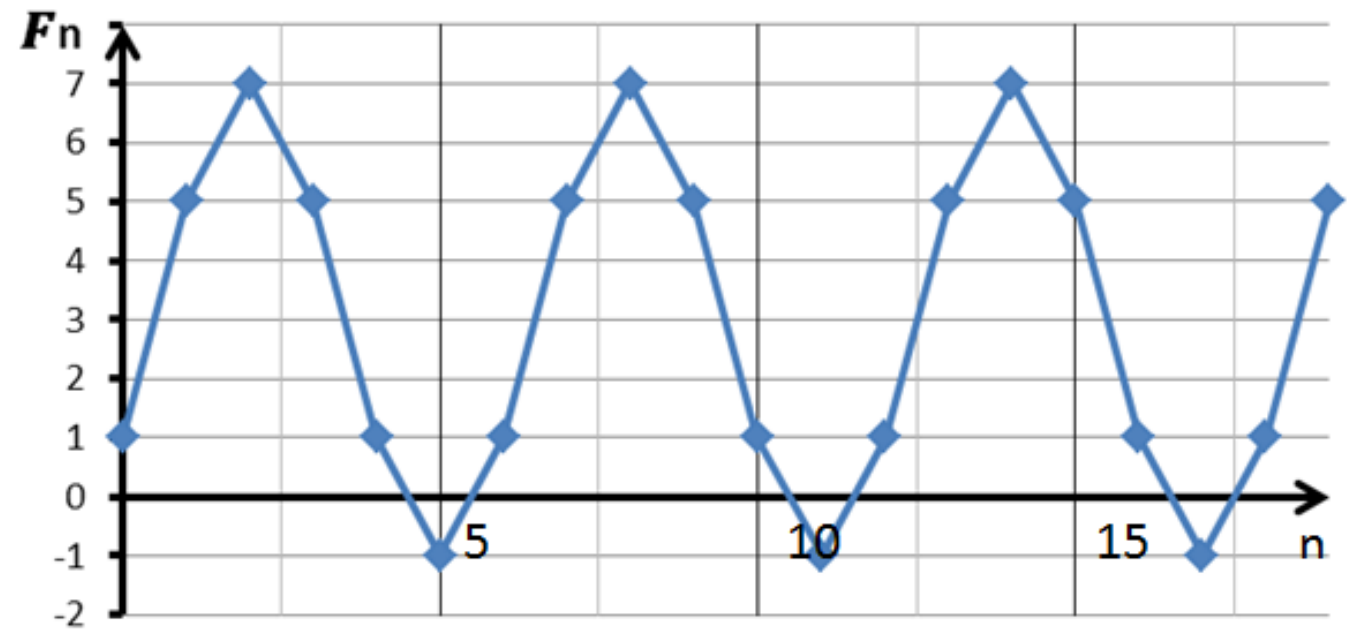

Figure 3. $F_{n}$ profile at $\mathrm{n}=0,1, \ldots 20 . F_{n+2}=3+F_{n+1}-F_{n}, n \geq 0, \quad F_{0}=1, \quad F_{1}=5 . T=6$

\section{Example 4}

Let $A=3, B=-1, C=-1, F_{0}=1, F_{1}=5$. Then $F_{n+2}=3-F_{n+1}-F_{n}, n \geq 0$. The profile of $F_{n}$ for $\mathrm{n}=1, \ldots 20$ is shown in Figure $5 . \mathrm{F}_{\mathrm{n}}, F_{n+2}=3-F_{n+1}-$ $F_{n}, n \geq 0$, is periodic with period $T=3$, as it follows from formulas $T=\frac{2 \pi}{\omega}, \omega=\operatorname{acos} \frac{B}{2}$.

For the first $n=0,1, \ldots 13$, the terms of $F_{n}$ are given in Table 4.
Table 4

\begin{tabular}{|c|c|c|c|c|c|c|c|}
\hline $\mathrm{n}=$ & 0 & 1 & 2 & 3 & 4 & 5 & 6 \\
\hline $\mathrm{F}_{\mathrm{n}}=$ & 1.0 & 5.0 & -3.0 & 1.0 & 5.0 & -3.0 & 1.0 \\
\hline $\mathrm{n}=$ & 7 & 8 & 9 & 10 & 11 & 12 & 13 \\
\hline $\mathrm{F}_{\mathrm{n}}=$ & 5.0 & -3.0 & 1.0 & 5.0 & -3.0 & 1.0 & 5.0 \\
\hline
\end{tabular}




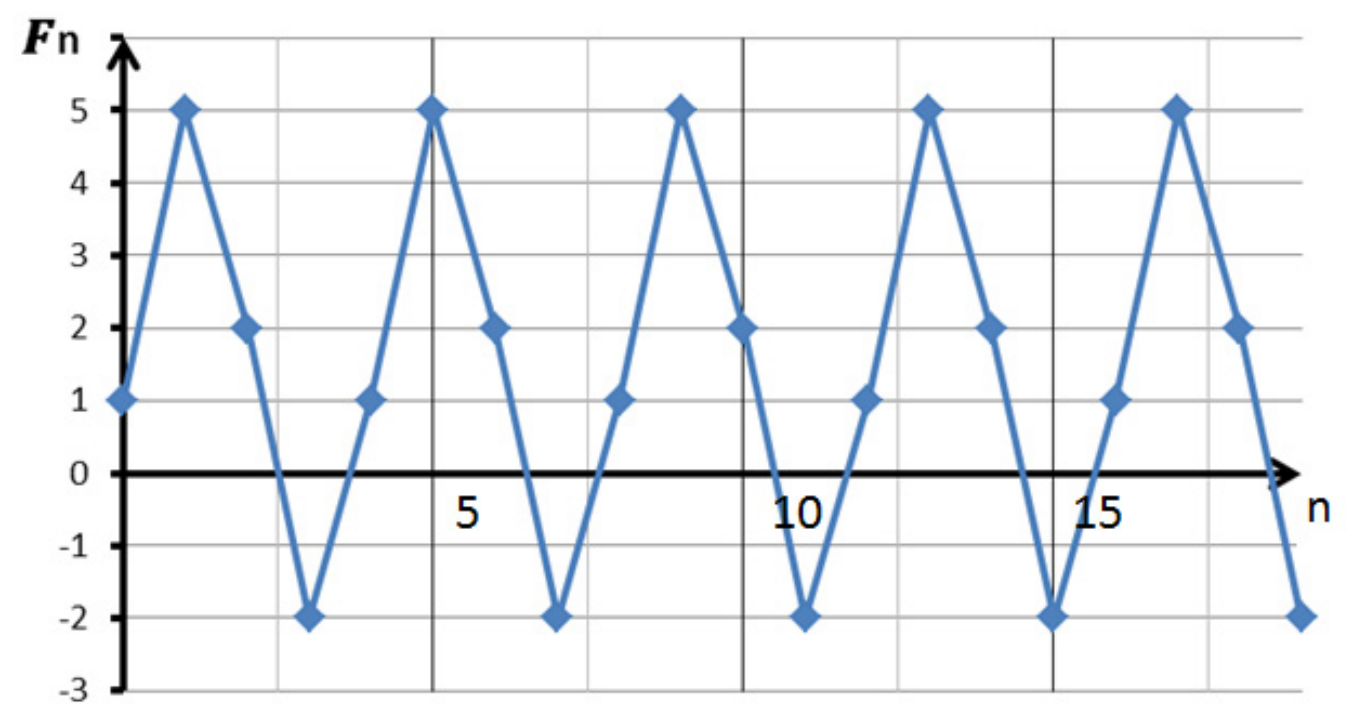

Figure 4. $\mathrm{F}_{\mathrm{n}}$ profile at $\mathrm{n}=0,1, \ldots 20 . F_{n+2}=3-F_{n}, n \geq 0, F_{0}=1, F_{1}=5 . T=4$

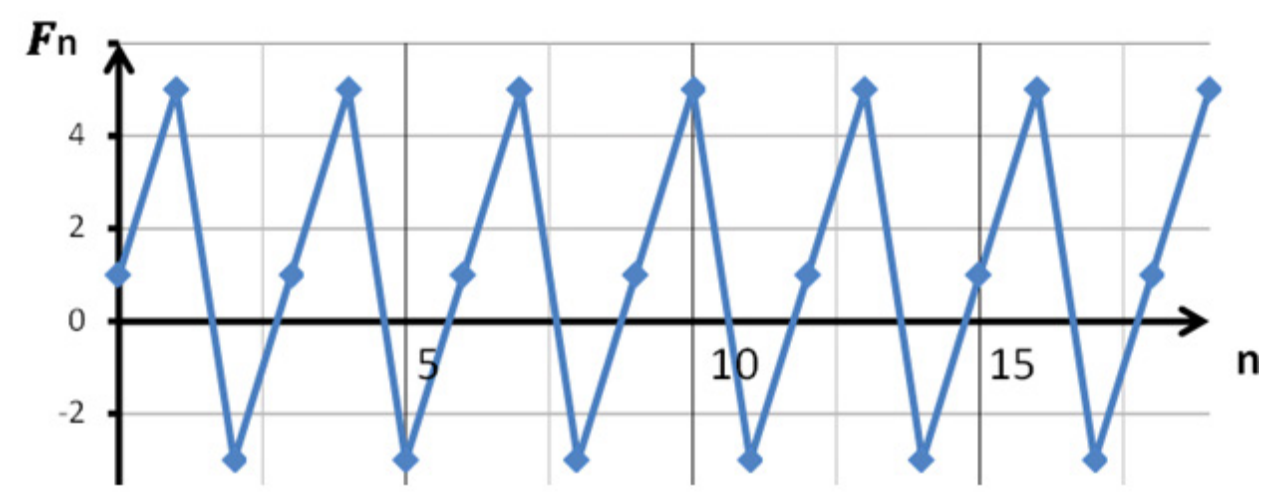

Figure 5. $\mathrm{F}_{\mathrm{n}}$ profile at $\mathrm{n}=0,1, \ldots 20 . F_{n+2}=3-F_{n+1}-F_{n}, n \geq 0 . \quad F_{0}=1, \quad F_{1}=5 . T=3$

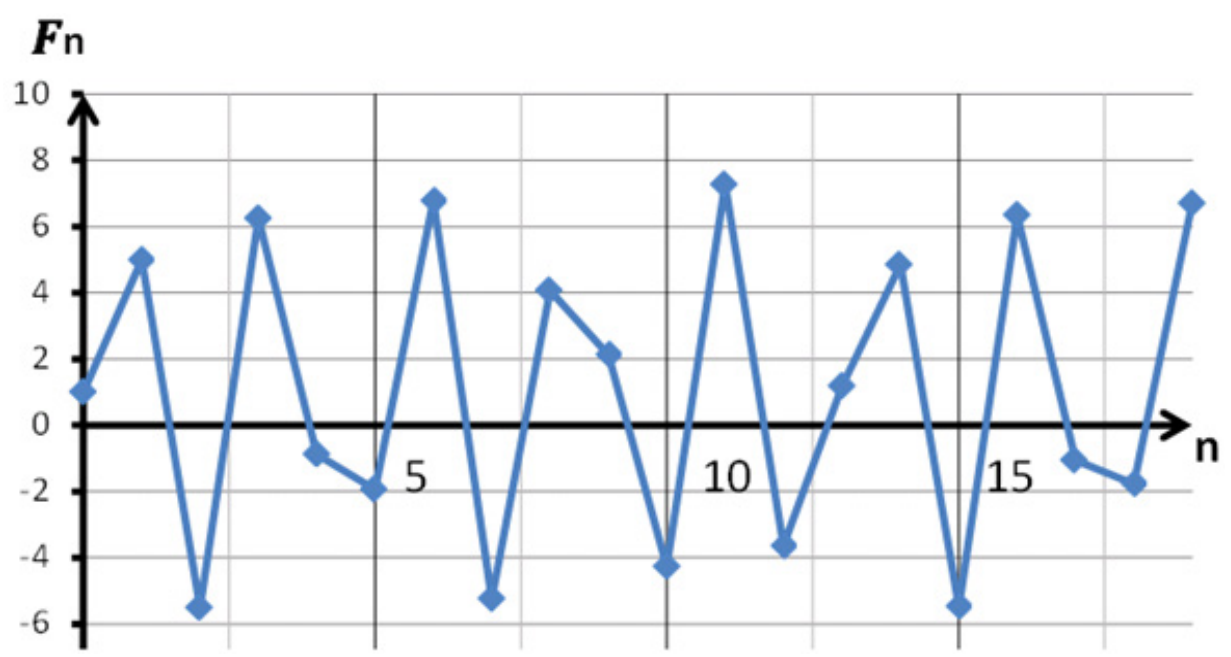

Figure 6. The profile of $\mathrm{F}_{\mathrm{n}}$ at $\mathrm{n}=0,1, \ldots 20 . F_{n+2}=3-1.5 F_{n+1}-F_{n}, n \geq 0, F_{0}=1, F_{1}=5 . T=2.6$

\section{Example 5}

Choose $A=3, B=-1.5, C=-1, F_{0}=1, F_{1}=5$. Then $F_{n+2}=3-1.5 F_{n+1}-F_{n}, n \geq 0$. In Figure 6 , we have plotted the sequence profile $\mathrm{F}_{\mathrm{n}}, F_{n+2}=3-$ $1.5 F_{n+1}-F_{n}, n \geq 0$, at $\mathrm{n}=1, \ldots 20$. The plot shows that $\mathrm{F}_{\mathrm{n}}$ is periodic with period $\mathrm{T}=2.51$. According to (12), $\omega=\operatorname{acos} \frac{B}{2}=\operatorname{acos} \frac{-1.5}{2}, \quad T=\frac{2 \pi}{\omega}=2.51$. For the first $\mathrm{n}=0,1, \ldots 13$, the terms of sequence $\mathrm{F}_{\mathrm{n}}$ are given in Table 5 .

Table 5.

\begin{tabular}{|c|c|c|c|c|c|c|c|}
\hline $\mathrm{n}=$ & 0 & 1 & 2 & 3 & 4 & 5 & 6 \\
\hline $\mathrm{F}_{\mathrm{n}}=$ & 1.0 & 5.0 & -5.5 & 6.3 & -0.9 & -1.9 & 6.8 \\
\hline $\mathrm{n}=$ & 7 & 8 & 9 & 10 & 11 & 12 & 13 \\
\hline $\mathrm{F}_{\mathrm{n}}=$ & -5.2 & 4.1 & 2.1 & -4.3 & 7.3 & -3.6 & 1.2 \\
\hline
\end{tabular}




\subsection{Numerical Examples of Solutions of the Wave Equation Containing Periodic Fibonacci-Like Sequences on Graphs}

As it was mentioned above, finite difference approximations of the PDE are based upon replacing partial differential equations by finite difference equations using Taylor approximations [18]. Consider a hyperbolic PDE with two spatial independent variables.

$$
\frac{\partial^{2} \mathrm{f}}{\partial \mathrm{t}^{2}}=\mathrm{a} \frac{\partial^{2} \mathrm{f}}{\partial \mathrm{x}^{2}}+\mathrm{b} \frac{\partial^{2} \mathrm{f}}{\partial \mathrm{y}^{2}}
$$

where $f=f(x, y, t), \quad a=a(x, y, t), \quad b=b(x, y, t), \quad g=$ $\mathrm{g}(\mathrm{x}, \mathrm{y}, \mathrm{t})$. Using a standard two-dimensional spatial orthogonal grid $\mathrm{H}$ with points $\mathrm{v}_{\mathrm{ps}}=(\mathrm{p} \Delta \mathrm{x}, \mathrm{s} \Delta \mathrm{y})$, the forward difference formula for the derivative with respect to $t$, and the central difference formula for the second derivatives with respect to $\mathrm{x}$ and $\mathrm{y}$, we obtain the following equivalent finite deference equation

$$
\begin{aligned}
& \frac{f_{i, j}^{n+1}-2 f_{i, j}^{n}+f_{i, j}^{n-1}}{\Delta t^{2}} \\
& =a_{i, j}^{n} \frac{f_{i-1, j}^{n}-2 f_{i, j}^{n}+f_{i+1, j}^{n}}{\Delta x^{2}}+b_{i, j}^{n} \frac{f_{i, j-1}^{n}-2 f_{i, j}^{n}+f_{i, j+1}^{n}}{\Delta y^{2}}
\end{aligned}
$$

where $\quad x=i \Delta x, y=j \Delta y, i, j=1,2, \ldots, t=n \Delta t . \quad$ By construction, grid $\mathrm{H}$ is a graph, in which point $(i, j)$ is adjacent to points $(i, j \pm 1)$ and $(i \pm 1, j)$. The nearest neighborhood (ball) of point $(i, j)$ is the subgraph $\mathrm{U}((i, j)$ containing $(i, j)$ and points $(i, j \pm 1)$ and $(i \pm 1, j)$. Using these notations, equation (13) can be written as the following.

$$
\mathrm{f}_{\mathrm{i}, \mathrm{j}}^{\mathrm{n}+1}=\sum_{(k, p) \in U(i, j)} \mathrm{c}_{\mathrm{i}, \mathrm{j}, \mathrm{k}, \mathrm{p}}^{\mathrm{n}} \mathrm{f}_{\mathrm{k}, \mathrm{p}}^{\mathrm{n}}+\mathrm{f}_{\mathrm{i}, \mathrm{j}}^{\mathrm{n}}-\mathrm{f}_{\mathrm{i}, \mathrm{j}}^{\mathrm{n}-1}, \mathrm{t}=\mathrm{n}
$$

where $\sum_{(\mathrm{k}, \mathrm{p}) \in \mathrm{U}(i, j)} \mathrm{c}_{\mathrm{pk}}^{\mathrm{n}}=1$.

The set (14) can be applied to a general graph $\mathrm{G}(\mathrm{V}, \mathrm{W})$ with the set of points $V=\left(v_{1}, v_{2}, \ldots v_{s}\right)$ and the set of edges $\mathrm{W}=\left(\left(\mathrm{v}_{\mathrm{p}} \mathrm{v}_{\mathrm{q}}\right), \ldots.\right)$, where $\mathrm{U}\left(\mathrm{v}_{\mathrm{p}}\right)$ is the ball of point $\mathrm{v}_{\mathrm{p}}$, $p=1, \ldots$. s. Set (14) is similar to the set of differential equations on a graph investigated by A. I. Volpert in [20].

The wave equation defined below is a special case of hyperbolic equation (14) if the following condition holds

$$
\begin{gathered}
\mathrm{f}_{\mathrm{p}}^{\mathrm{n}+1}=\sum_{v_{k} \in U\left(v_{p}\right)} \mathrm{c}_{\mathrm{pk}}^{\mathrm{n}} \mathrm{f}_{\mathrm{k}}^{\mathrm{n}}+\mathrm{f}_{\mathrm{p}}^{\mathrm{n}}-\mathrm{f}_{\mathrm{p}}^{\mathrm{n}-1}, \mathrm{t}=\mathrm{n} \\
\sum_{v_{k} \in U\left(v_{p}\right)} \mathrm{c}_{\mathrm{pk}}^{\mathrm{n}}=1, c_{p k}^{n}=c_{p k} \geq 0, \mathrm{t}=\mathrm{n}, \mathrm{p}=1, \ldots \mathrm{s} .
\end{gathered}
$$

The solution of this equation should be analogous the solution of the wave equation in the continuous case. It should be associated with oscillations of the function at points of $\mathrm{G}$ and the wave propagation.

There is considerable amount of literature devoted to the study of different approaches to digital lines, surfaces and spaces used by researchers. Traditionally, a digital image has a graph structure (see $[2,3,5])$. A digital space $G$ is a simple undirected graph $G=(V, W)$ where $\mathrm{V}=\left(\mathrm{v}_{1}, \mathrm{v}_{2}, \ldots \mathrm{v}_{\mathrm{n}}, \ldots\right)$ is a finite or countable set of points, and
$\mathrm{W}=\left(\left(\mathrm{v}_{\mathrm{p}} \mathrm{v}_{\mathrm{q}}\right), \ldots.\right)$ is a set of edges. The induced subgraph $U(v)$ containing point $\mathrm{v}$ and all points adjacent to $\mathrm{v}$ is called the ball of point $v$ in $G$. Graphs that are digital counterparts of continuous n-dimensional manifolds were studied in $[5,6,7,8]$. Consider the numerical solutions of the initial value problem for the wave equation on a graph $\mathrm{G}\left(\mathrm{v}_{1}, \mathrm{v}_{2}, \ldots \mathrm{v}_{\mathrm{s}}\right)$. We will see that periodic Fibonacci-like sequences are basic elements of this equation.

Example 6. Numerical solution of the initial value problem on a connected graph $G$ with two points.

Consider a connected graph $G\left(v_{1}, v_{2}\right)$ consisting of two adjacent points depicted in Figure 7(a). According to (15)

$$
\left\{\begin{array}{l}
f_{1}^{n+1}=c_{11} f_{1}^{n}+c_{12} f_{2}^{n}+f_{1}^{n}-f_{1}^{n-1} \\
f_{2}^{n+1}=c_{21} f_{1}^{n}+c_{22} f_{2}^{n}+f_{2}^{n}-f_{2}^{n-1}
\end{array} .\right.
$$

Let $f_{1}^{0}+f_{2}^{0}=d$ and $f_{1}^{1}+f_{2}^{1}=d$. Then $f_{1}^{n}+f_{2}^{n}=d$ for any $\mathrm{n}>1$. For set (16) we have two sequences

$$
\left\{\begin{array}{l}
f_{1}^{n+1}=c_{12} d+\left(1+c_{11}-c_{12}\right) f_{1}^{n}-f_{1}^{n-1} \\
f_{2}^{n+1}=c_{21} d+\left(1+c_{22}-c_{21}\right) f_{2}^{n}-f_{2}^{n-1}
\end{array} .\right.
$$

According to theorem 3, sequence (17) is the generalized Fibonacci-like sequence with $A=c_{12} d, B=$ $1+c_{11}-c_{12}, C=-1$. Since $c_{11}+c_{21}=1, c_{21}+c_{22}=$ 1 , and $c_{p k}^{n} \geq 0$ then $|B|<2$. Therefore, sequence (17) is periodic with period $T=\frac{2 \pi}{\omega}>2, \omega=\arccos \frac{B}{2}=$ $\arccos \frac{1+c_{11}-c_{12}}{2}$ as it follows from (12). For the same reason, $f_{2}^{n+1}$ is periodic with the same period T. Define coefficients $c_{p k}$ in equation (16) in the following way; $c_{11}=0.9, c_{21}=0.1, c_{22}=0.9, c_{12}=0.1$. Initial values are given as $\mathrm{f}_{1}^{0}=\mathrm{f}_{1}^{1}=3, \mathrm{f}_{2}^{0}=\mathrm{f}_{2}^{1}=0, d=3$. Therefore, $f_{1}^{n+1}=0.3+1.8 f_{1}^{n}-f_{1}^{n-1}, f_{2}^{n+1}=0.3+1.8 f_{2}^{n}-f_{2}^{n-1}$.

The period of oscillations is $T=13.9$. The solution of the initial value problem at points 1 and 2 for $t=1, \ldots 50$ is displayed in Figure 7(b), which illustrates the time behavior of the values of function $f$. Figure 7(b) presents oscillations at points 1 and 2 .

Example 7. Numerical solution of the initial value problem on the digital Klein bottle.

A Klein bottle is an object of investigation in many fields. In physics [12], a Klein bottle attracted attention in studying lattice models on non-orientable surfaces A digital 2D Klein bottle $\mathrm{K}$ depicted in Figure 8(a) consists of sixteen points. The nearest neighborhood $\mathrm{O}\left(\mathrm{v}_{\mathrm{k}}\right)$ of every point $\mathrm{v}_{\mathrm{k}}$ is a digital 1-sphere containing six points. Topological properties of $\mathrm{K}$ are similar to topological properties of its continuous counterpart. For example, the Euler characteristic and the homology groups of a continuous and a digital Klein bottle are the same ([7] and [8]). Consider the solution of equation (15) on K. One can check directly that all sequences in set (15) are periodic generalized Fibonacci-like sequences. Define coefficients $c_{p k}$ in the following way. If points $\mathrm{v}_{\mathrm{p}}$ and $\mathrm{v}_{\mathrm{k}}$ are adjacent then $\mathrm{c}_{\mathrm{pk}}=0.01$ and $\mathrm{c}_{\mathrm{kk}}=0.94$ for $\mathrm{k}=1, \ldots 16$. Initial values are given as $\mathrm{f}_{6}^{0}=3, \mathrm{f}_{6}^{1}=3$. In Figure 8(b), numerical solutions at points 1 and 3 of the of the initial value problem are plotted at time $\mathrm{t}=1, \ldots 100$. Two lines show oscillations with period $\mathrm{T}>2$. 


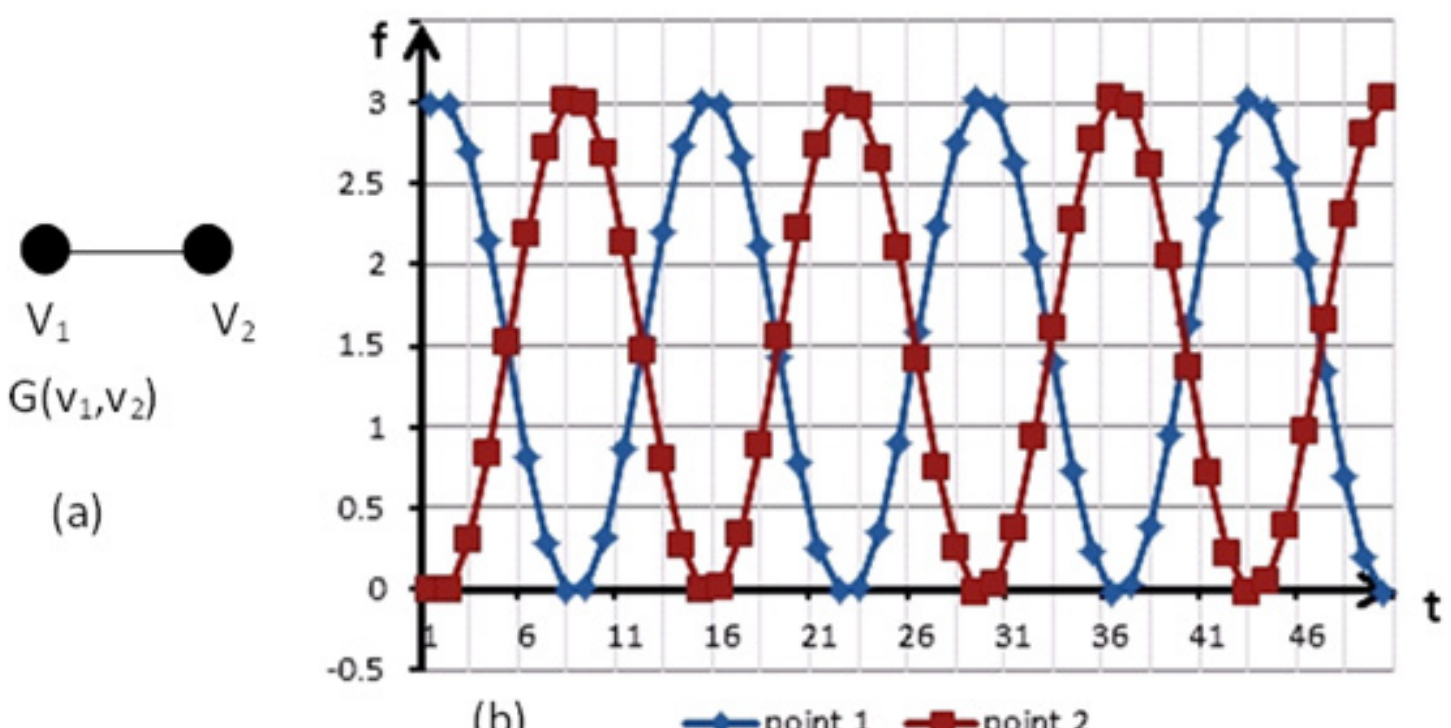

(b)

Figure 7. (a) $\mathrm{G}$ is a graph with two adjacent points. (b) Numerical solution of the initial value problem on graph $\mathrm{G}$ with two points $\mathrm{v}_{1}$ and $\mathrm{v}_{2}$. Initial values are $\mathrm{f}_{1}{ }^{0}=\mathrm{f}_{1}{ }^{1}=3, \mathrm{f}_{2}{ }^{0}=\mathrm{f}_{2}{ }^{1}=0$. The solutions on $\mathrm{G}$ are shown at points 1 and $2, \mathrm{t}=0,1, \ldots 50$, The solution profiles are oscillations with period $\mathrm{T}=13.9$

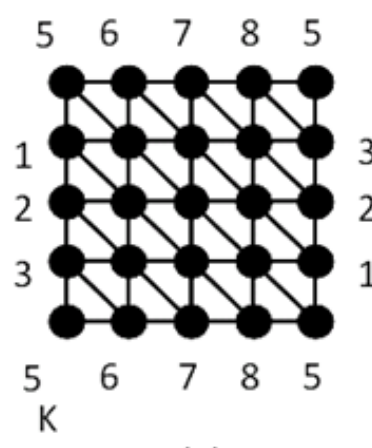

(a)

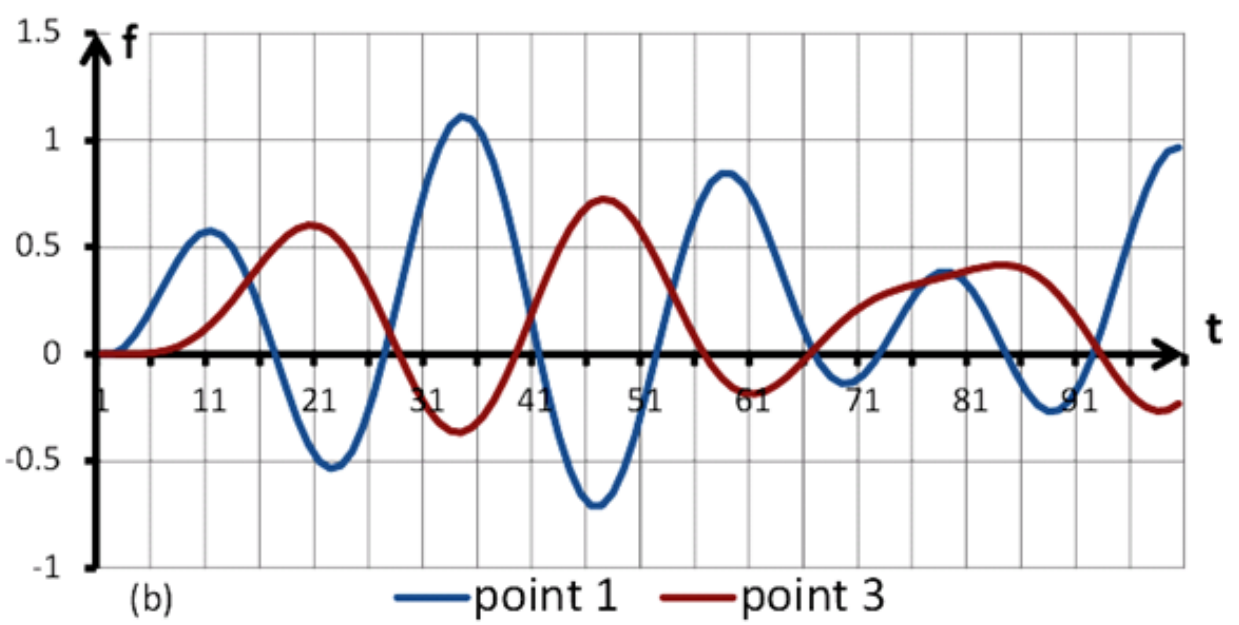

Figure. 8. (a) $\mathrm{K}$ is a digital Klein bottle. (b) The solution profiles of the initial value problem on the Klein bottle $\mathrm{K}$ at points 1 and $3, \mathrm{t}=0,1, \ldots 100$, $\mathrm{f}_{6}{ }^{0}=\mathrm{f}_{6}{ }^{1}=3, \mathrm{f}_{\mathrm{k}}{ }^{0}=\mathrm{f}_{\mathrm{k}}{ }^{1}=0, \mathrm{k} \neq 6$. Two lines are oscillations with period $\mathrm{T}>2$

\section{Conclusion}

In connection with computational methods for approximating the solutions of partial differential equations, the generalized Fibonacci-like sequence $F_{n+2}=A+B F_{n+1}+C F_{n}$ with $F_{0}=a, F_{1}=b, n \geq 0$, where $A, B, C, a$ and $b$ are real numbers is investigated. It is shown that if $|B|<2, C=-1$ then $\left\{F_{n}\right\}$ is periodic with the period $T>2$. If period $T=1$, then

$$
A=C=0, B=1, F_{n+1}=F_{n}, n \geq 0, F_{0}=F_{1}=a \text {. }
$$

If period $T=2$ and $C \neq 1$ then $B=C-1, D=\frac{A}{1-C}$, $F_{n+1}=D-F_{n}, n \geq 0, F_{0}=a, F_{1}=D-a$. Numerical examples of periodic Fibonacci-like sequences, and periodic numerical solutions of the wave equation on graphs are presented.

\section{Competing Interests}

The author declares that he has no competing interests.

\section{Acknowledgements}

The author has got no financial or any other support from any person, fund, organization, etc.

\section{References}

[1] Badshah, V.H., Teeth, M.S. and Dar, M.M., 'Generalized Fibonacci-Like Sequence and its Properties", Int. J. Contemp. Math. Sciences,7(24) (2012) 1155-1164.

[2] Bai, Y., Han, X., Prince, J., Digital Topology on Adaptive Octree Grids. Journal of Mathematical Imaging and Vision. 34(2) (2009) 165-184.

[3] Eckhardt, U. and Latecki, L., Topologies for the digital spaces Z2 and Z3. Computer Vision and Image Understanding, 90 (2003) 295-312.

[4] Evako, A., "Solution of a Parabolic Partial Differential Equation on Digital Spaces: A Klein Bottle, a Projective Plane, a 4D Sphere and a Moebius Band", International Journal of Discrete Mathematics, http://www.sciencepublishinggroup.com/j/dmath, in press

[5] Evako, A., Kopperman, R. and Mukhin, Y., Dimensional properties of graphs and digital spaces. Journal of Mathematical Imaging and Vision, 6 (1996) 109-119. 
[6] Evako, A., Classification of digital n-manifolds. Discrete Applied Mathematics. 181 (2015) 289-296.

[7] Ivashchenko, A., Representation of smooth surfaces by graphs. Transformations of graphs which do not change the Euler characteristic of graphs. Discrete Mathematics, 122 (1993) 219-233.

[8] Ivashchenko, A., Contractible transformations do not change the homology groups of graphs. Discrete Mathematics, 126 (1994) $159-170$

[9] Falcon, S. and Plaza, A.," k-Fibonacci sequences modulo m", Chaos, Solitons and Fractals", 41 (2009) 497-504.

[10] Koshy, T., Fibonacci and Lucas Numbers with Applications, Wiley, New York, 2001.

[11] Li, H-C., " Complete and reduced residue systems of second-order recurrences modulo p", Fibonacci Quart., 38 (2000) 272-281

[12] $\mathrm{Lu}, \mathrm{W} . \mathrm{T}$. and $\mathrm{Wu}, \mathrm{F}$. Y., Ising model on nonorientable surfaces: Exact solution for the Moebius strip and the Klein bottle. Phys. Rev., E 63 (2001) 026107.

[13] Morton, K.W. and Mayers, D.E., Numerical Solution of Partial Differential Equations, An Introduction. Cambridge University Press, 2005.

[14] Pokornyi, Y. and Borovskikh, A., "Differential equations on networks (Geometric graphs)", Journal of Mathematical Science, 119 (6) (2004) 691-718.

[15] Schork, M., "Generalized Heisenberg algebras and k-generalized Fibonacci numbers", J. Phys. A: Math. Theor., 40 (2007). 4207-4214.

[16] Shu, T.L. and Chen, G., "Oscillations of Second-Order Nonlinear Partial Difference Equations", Rocky Mountain J. Math., 34(2) (2004) 699-711.

[17] Singh, M., Gupta, Y.K. and Sikhwal, O., "Generalized Fibonacci Lucas sequence its Properties", Global Journal of Mathematical Analysis, 2 (3) (2014) 160-168.

[18] Smith, G.D., Numerical solution of partial differential equations: finite difference methods (3rd ed.), Oxford University Press, 1985.

[19] Tuwankotta, J.M.," Two aspects of a generalized Fibonacci sequence", J. Indones. Math. Soc., 21(1) (2015). 1-17.

[20] Vol'pert, A., Differential equations on graphs. Mat. Sb. (N. S.), 88 (130) (1972) 578-588

[21] Wall, D.D., "Fibonacci series modulo m", Amer. Math. Monthly, 67 (1960).525-532.

[22] Walton, J.E. and Horadam, A.F., "Some Aspects of Generalized Fibonacci Sequence", The Fibonacci Quaterly, 12 (3) (1974). 241-250. 